Project 90304

\title{
Acoustic Monitor for Liquid-Solid Slurries Measurements at Low Weight Fractions
}

\author{
Tavlarides, Lawrence L.
}

Syracuse University

\section{RESULTS TO DATE: INTRODUCTION}

Our effort in this project is to develop an acoustic monitor for accurate, real-time characterization of the size and weight fractions of solids in slurries for process monitoring and to determine the optimal timing for slurry transfers. This capability will be valuable in the Savannah River Site accelerated clean-up program. Our scientific work during the first research period developed a theory, supported by experiments, to describe sound attenuation of solids in suspensions in the presence of bubbles, which permits us to determine the solid-liquid weight percent. Engineering developments during the second research period led to the design, construction, and demonstration, in our laboratories, of the Syracuse Acoustic Monitor (SAM) system that measures weight percent solids accurately in slurries of 0.5 to 8.0 weight percent online and in real-time. Also, we had shown the potential for these measurements in solid-gas-liquid slurries by removing the interference due to the presence of gas bubbles.

\section{PROPOSED WORK}

The proposed work for the current research period includes the following elements: 1) refinement of the software for accurate and rapid data analyses of solid-gas-liquid slurries; 2) development of a metal (or ceramic) housing for the monitor; 3) demonstration of the acoustic monitor for SRS surrogate slurries in our laboratory; 4) testing of the redesigned unit in the Filtration Research Engineering Demonstration(FRED) pilot plant facility at the University of South Carolina; and 5) modification of the acoustic monitor to a more compact pulse-echo configuration for potential in-tank placement.

\section{PROGRESS}

We have made substantial progress on the first three elements of the project and will complete them by December 14, 2004 as specified in the proposal for this funding period.

Software refinement for rapid data analysis of solid-gas-liquid slurries: The first generation software designed to control the acoustic monitor operations, perform data acquisition operations and execute calculations to obtain real-time concentration readings has been modified and updated to achieve higher performance and required accuracy when using our ?bubble elimination? method.

Modifications include: a) Inclusion of on-line calculations to determine the minimal necessary number of measurements required to obtain concentration readings with the pre-established accuracy and confidence interval. b) Simultaneous execution of different processes such as data acquisition and data processing (spectral and statistical) for the previous operations. c) Optimization of the signal acquisition process which reduces the number of iterations of signal captures to secure optimal parameter values. d) Flexibility of software to independently vary the number of pulses/receptions of each channel (currently six). e) Signal processing developed to correctly detect working frequencies of ultrasonic transducers in the presence of extension cables (100 feet), which distort the characteristics of the transmitted signals.

The revised software produces stable real-time results at 3.1 milliseconds response time (versus $4 \mathrm{~ms}$ ) and full measurement cycle of 0.1 to $0.2 \mathrm{~s}$ versus 0.5 to $1.0 \mathrm{~s}$ in the presence of gas bubbles with extension cables of 100 feet between monitor and computer. 
Development of a metal (or ceramic) housing for the monitor: The original in-line acoustic monitor is constructed of an acrylic material with acoustic impedances similar to the slurry and water. Other housing materials were evaluated for a) acceptable radiation and chemical stability to minimize maintenance, b) appropriate acoustic properties of transparency in the required frequency range and acoustic impedance to match the transducers and slurry, c) adequate mechanical properties to prevent fracture of the housing structure for sustained operation.

Materials considered to meet all these requirements include steel, ceramics, acrylic resins, and high strength plastics such as Ultem 1000 and PEEK 1000 (trademarks of G.E. Plastics and Victrex plc respectively). Ultem 1000, demonstrated the best overall results and was chosen for construction of the in-line test cell. Although the material displays good tensile and compression strength, a new design of the housing was developed to diminish crack occurrence. The new cell has been tested and displays the desirable acoustic properties of the original housing while providing acceptable radiation and chemical stability as well as higher stress resistance to protect transducers and minimize maintenance.

Demonstration of the acoustic monitor for SRS surrogate slurries in our laboratories: This activity is in progress and will be completed in the remaining three months of this funding period. We have recently received adequate samples of Tank No. 8 surrogate sludge and supernate from SRS provided by Messieurs Samuel Fink and Michael Poirier of SRS, September 8, 2004. These materials will permit laboratory testing and calibrations to be made for both the sludge and sludge with monosodium titanate (MST) or permanganate. This work was delayed until the second generation monitor with acceptable housing materials is available.

At the close of this funding period we will have achieved our stated objectives. 